\title{
The effect of bottom boundary conditions in the ice-sheet to ice-shelf transition zone problem
}

\author{
Alexander V. WILCHINSKY \\ Centre for Polar Observation and Modelling, Department of Earth Sciences, University College London, Gower Street, \\ London WC1E 6BT, UK \\ E-mail: aw@cpom.ucl.ac.uk
}

\begin{abstract}
Uniqueness of the transition zone solution is discussed. It is argued that when it is assumed that the stresses are continuous at the grounding line, the ice-shelf solution at the grounding line should possess a zero slope. In order to avoid issues caused by the mathematical singularity of the solution, the same technique as that used to study the transition zone is applied to a similar problem of lifting an elastic sheet from a rigid substrate, which allows a better physical understanding. This exemplifies the effect of the bottom boundary condition of no vertical motion imposed up to a fixed grounding line position, while the forcing parameters, such as the flow rate, vary. Its effect is to produce multiple solutions due to suppression of the separation mechanism.
\end{abstract}

\section{INTRODUCTION}

Ice-sheet motion is determined by the transition zone dynamics that couple the grounded part of an ice sheet to its floating part, the ice shelf. As the shallow-ice approximation makes equations governing the grounding part of the ice sheet simpler, it is convenient to decouple the grounded icesheet problem from the ice-shelf problem. This leads to the necessity of imposing boundary conditions at the grounding line. Although it has been argued that, due to its low stress, the presence of the ice shelf does not constrain the ice-sheet dynamics in any way apart from the condition of hydrostatic equilibrium at the grounding line (Hindmarsh, 1993), here we discuss the other possibility, namely that the ice-sheet motion is uniquely determined by its external and initial conditions. The latter implies two boundary conditions at the grounding line that, together with the condition of zero mass flux at the ice divide, close the isothermal ice-sheet problem described by a non-linear parabolic second-order differential equation solved between the ice divide and the unknown grounding line position.

To date, no work has been able to show uniqueness of the transition zone solution that, apart from the hydrostatic equilibrium condition, would allow us to impose an additional unique boundary condition at the grounding line to relate the ice thickness to the mass flow rate. The progress in doing so has been hindered by the complexity of the problem, especially due to a singularity of the solution at the grounding line caused by a jump in the bottom boundary conditions.

Chugunov and Wilchinsky (1996) used a sigma transformation of the coordinate system and finite differences to solve the ice-sheet to ice-shelf transition problem in its steady-state form. However, in order to find a constant that, firstly, for non-linear rheology with $n$ being the Glen's flowlaw exponent, can be written as (Wilchinsky and Chugunov, 2001)

$$
\beta \propto h\left(x_{\mathrm{g}}\right)^{(n+2) / n} / q\left(x_{\mathrm{g}}\right)^{1 / n}
$$

and, secondly, appears when both the mass flow rate, $q$, and the thickness, $h$, at the grounding line $\left(x=x_{\mathrm{g}}\right)$ are normalized to unity in the transition zone, they had to assume that the sought ice-shelf bottom is horizontal at the grounding line. The resulting magnitude of $\beta$ then determines the sought, unique relationship between the ice thickness and flow rate at the grounding line that constitutes the second boundary condition. The necessity to make an assumption that the ice-shelf bottom is horizontal at the grounding line was justified neither physically nor mathematically. Without this assumption the solution of the transition problem could be generally found for an arbitrary ice-shelf bottom slope and a fixed ice thickness at the grounding line, that could lead to the conclusion that there is no unique connection between the mass flow rate and the ice thickness at the grounding line.

Although the problem of uniqueness of the transition zone solution could be solved eventually using rigorous mathematical analysis, here we aim at finding some insights into the issue that can be drawn through an intuitive understanding. In particular, we consider the similar problem of lifting an elastic sheet with an unknown lift line that, when solved using the same technique as the transition zone problem, produces similar results but allows a better physical understanding. Our purpose here is to outline a way which more detailed studies can follow in order to find the final answer to the problem, as any, even small, progress in understanding this contentious problem should be made available to ice-sheet modellers for further discussion. In particular, here we argue that the possibility of multiple solutions of the transition zone problem may be due to the specific way of posing the mathematical problem at the grounding line that prevents separation of the ice from the bedrock. We also show that if the physical interaction between the grounded ice and the water in the vicinity of the grounding line is taken into account, then a unique relationship between the mass flow rate and the ice thickness at the grounding line should exist.

\section{THE GROUNDING LINE POSITION}

The problem of marine ice-sheet dynamics requires different boundary conditions at the bottom of the grounded part and the floating part separated by the grounding line, $x_{\mathrm{g}}$ (Fig. 1). We consider two-dimensional flow of an ice sheet. At the grounded ice-sheet bottom $(z=b$, where $b$ is the ice-shelf bottom elevation; $x<x_{\mathrm{g}}$ ) that is considered here to be flat 


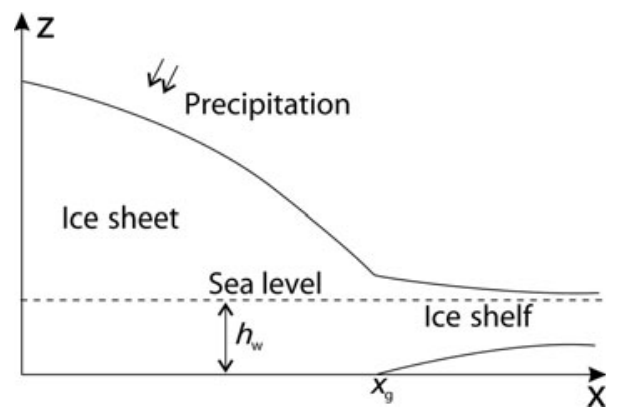

Fig. 1. Schematic of a marine ice sheet.

and horizontal for simplicity, a sliding law is applied together with an assumption of negligible melting

$$
\begin{array}{rl}
v_{x}=k \tau_{x z^{\prime}}^{m} & x<x_{\mathrm{g}}, \\
v_{z}=0, & x<x_{\mathrm{g}},
\end{array}
$$

where $(x, z)$ are the horizontal and vertical coordinates, $v_{x}$ and $v_{z}$ are horizontal and vertical velocities, $\tau_{x z}$ is the bottom shear stress, $k$ is a friction coefficient and $m$ is an exponent. The condition $k=0$ yields a no-slip condition at the bottom. Some significant sliding can be present due to complex basal processes in the transition zone, and the noslip condition is used purely for mathematical simplicity, in order to represent the problem in its most basic form (Lestringant, 1994; Chugunov and Wilchinsky, 1996).

At the ice-shelf bottom, stress continuity yields

$$
\begin{gathered}
\frac{\mathrm{d} b}{\mathrm{~d} x} \sigma_{x z}-\sigma_{z z}=h_{\mathrm{w}} \rho_{\mathrm{w}} g, \\
\sigma_{x z}-\frac{\mathrm{d} b}{\mathrm{~d} x} \sigma_{x x}=\frac{\mathrm{d} b}{\mathrm{~d} x} h_{\mathrm{w}} \rho_{\mathrm{w}} g, \quad x>x_{\mathrm{g}},
\end{gathered}
$$

where $b$ is the ice-shelf bottom elevation, $h_{\mathrm{w}}$ is the water depth, $\rho_{\mathrm{w}}$ is the water density, $g$ is gravity acceleration and $\sigma_{z z}$ and $\sigma_{x z}$ are the corresponding components of the full stress tensor. The grounding line position, $x_{g}$ is not a prescribed magnitude, but is determined by the dynamics of the lower surface of the ice. In particular, if the ice-shelf bottom sinks up to the bedrock, the grounding line advances. If the sea water can penetrate and lift the grounded ice in the vicinity of the grounding line, the grounding line retreats. From this it is clear that the ice sheet stays grounded when the water pressure, $h_{w} \rho_{w} g$, is smaller than the stress with which the ice pushes downwards along the normal, $\mathbf{n}$, to its surface, $-\mathbf{n} \cdot \boldsymbol{\sigma} \cdot \mathbf{n}$, so that the sea water cannot penetrate under the ice sheet:

$$
-\mathbf{n} \cdot \boldsymbol{\sigma}(b) \cdot \mathbf{n}=-\sigma_{z z}(b)>h_{\mathrm{w}} \rho_{\mathrm{w}} g, \quad x<x_{\mathrm{g}} .
$$

At the same time at the ice-shelf bottom these stresses are equal:

$$
-\mathbf{n} \cdot \boldsymbol{\sigma}(b) \cdot \mathbf{n}=h_{\mathrm{w}} \rho_{\mathrm{w}} g, \quad x>x_{\mathrm{g}},
$$

that is, effectively Equation (4). Therefore in general terms the grounding line position is effectively determined by two conditions at the ice bottom

$$
\begin{aligned}
-\mathbf{n} \cdot \boldsymbol{\sigma}(b) \cdot \mathbf{n}=h_{\mathrm{w}} \rho_{\mathrm{w}} g, & x=x_{\mathrm{g}}+, \\
{[\mathbf{n} \cdot \boldsymbol{\sigma}(b) \cdot \mathbf{n}]_{x=x_{\mathrm{g}}}>0, } & x=x_{\mathrm{g}}-,
\end{aligned}
$$

where the brackets denote a jump while the + and - signs denote the limits from the right and left to $X_{g}$ respectively. If we now abstract, for a moment, from the issues associated

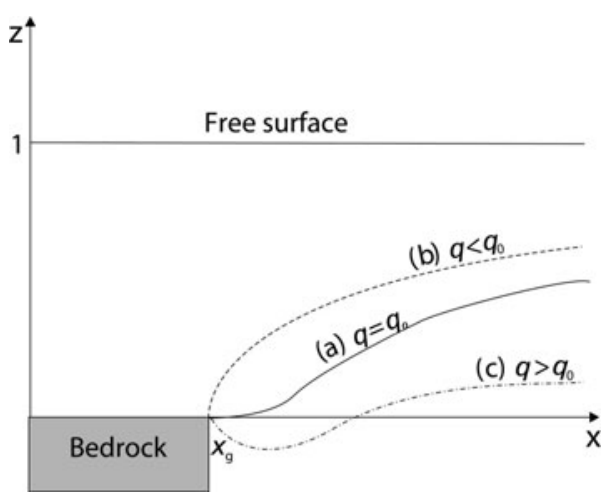

Fig. 2. Different cases of the ice-shelf bottom elevation, $b$, for a fixed ice thickness at the grounding line: (a) a zero slope at the grounding line is determined by the integral mass flow rate, $q$, equal to $q_{0}$; (b) a positive slope is determined by $q<q_{0}$; and (c) a negative slope is determined by $q>q_{0}$.

with the singularity of the mathematical problem, and make the realistic physical assumption that the stress is continuous across the grounding line in real ice sheets, then Equations (8) and (4) yield

$$
\frac{\mathrm{d} b}{\mathrm{~d} x}=0, \quad x=x_{\mathrm{g}}
$$

so the shelf slope at the grounding line is horizontal.

It should be noted that conditions (8) and (9) or (10) require knowledge of the stress or the shelf bottom profile in the transition zone. Therefore they are not convenient to use in the ice-sheet models written in terms of the shallow-ice approximation. One therefore strives to determine conditions for the mass flow rate and the ice thickness at the grounding line that could determine the grounding line position. In order to do this the ice flow in the ice-sheet to ice-shelf transition zone must be considered.

\section{THE TRANSITION ZONE PROBLEM}

Here we summarize main features of the transition zone solution that were described by Wilchinsky and Chugunov (2001), where more details can be found. A no-slip condition was used at the bedrock, and a perturbation analysis allows us to reduce the transition zone problem to solution of the full Stokes equations in a domain with a flat upper surface coinciding with the sea level to order $\delta=\left(\rho_{\mathrm{w}}-\rho_{\mathrm{i}}\right) / \rho_{\mathrm{w}} \approx 0.1$, where $\rho_{\mathrm{i}}$ is the density of ice (Fig. 2). The sea bottom was approximated to be flat, while the ice-shelf bottom is an unknown surface. A special nondimensionalization ensured that the mass flow rate and the grounding line thickness are both unity, which leads to the appearance of the parameter $\beta$ in the shelf thickness equation. The grounding line position is fixed. Variation of $\beta$ in this case is analogous to variation of the mass flow rate, $q\left(x_{\mathrm{g}}\right)$, when the ice thickness (or water depth) is fixed. Because of the fixed grounding line position, such a variation of $\beta$ is not allowed to affect the grounding line position. Because of this, the only way to determine whether a solution of the transition zone problem with a fixed grounding line is realistic is to check whether the conditions determining the grounding line, (8) and (9) or (10), hold.

Typical ice-shelf bottom profiles for different mass flow rate values, $q\left(x_{g}\right)$, are presented schematically in Figure 2, 
generated during the numerical analysis described above. This effect of the variation in mass flow rate is supported by recent finite-element simulations (S. Nowicki and D. Wingham, unpublished information). It can also be inferred using much simpler arguments. If we consider a constant flow rate solution of an ice shelf of unit thickness at the grounding line, found using the shallow-ice approximation (MacAyeal and Barcilon, 1988)

$$
h=[1+(\alpha+1) x / q]^{-1 /(n+1)},
$$

where $x$ is the scaled distance from the grounding line, then $h^{\prime}(0)=-1 / q$. The limits of the idealized ice-shelf solution, $h^{\prime}(0) \rightarrow 0$ as $q \rightarrow \infty$ and $h^{\prime}(0) \rightarrow-\infty$ as $q \rightarrow 0$, reflect the general behaviour of such a transition zone solution: when the ice thickness is fixed, as the mass flow rate decreases the bottom slope becomes more positive; and as the mass flow rate increases the ice-shelf bottom slope becomes more negative (Fig. 2). Furthermore, within the transition zone, the effect of the no-slip condition used in its grounded part is still significant, so the horizontal velocity increases with distance from the bottom. This implies that the shear stress in the ice shelf is non-negative, and Equation (4) shows that the stress, $-\sigma_{z z \text {, }}$ acting on a horizontal plane from above is larger than the hydrostatic stress when $\mathrm{d} b / \mathrm{d} x<0$ (negative bottom slope) and smaller than the hydrostatic stress when $\mathrm{d} b / \mathrm{d} x>0$ (positive bottom slope). The situation with a negative bottom slope clearly shows that the ice sheet would advance.

The simple condition for the shelf bottom slope Equation (10) was derived here assuming continuity of the stresses at the grounding line. It was also used by Chugunov and Wilchinsky (1996) in order to determine $\beta$ in Equation (1), which provides the sought relationship between the mass flow rate and the ice thickness at the grounding line. The latter was done without any justification, and it is now clear that it is tantamount to requiring that the stress is continuous at the grounding line. A unique $\beta$ makes it possible to identify different grounding line positions, for example, by varying the water depth (Fig. 3). However, the assumption of continuity can be questioned, as a problem describing a jump between a no-slip condition and a slip condition yields infinite stresses (Barcilon and MacAyeal, 1993). Without this assumption one has to use more general conditions (Equations (8) and (9)). Because of the singularity at the grounding line, these conditions may be satisfied for a range of $\beta$, determining a range of non-zero angles of the shelf bottom slope (S. Nowicki and D. Wingham, unpublished information). In this case multiple solutions may exist. One should, however, take into consideration that the described jump in the boundary condition makes the continuum mechanical model invalid at the jump itself where the strain rates are discontinuous and stresses are infinite. Because of this, it is not clear if one can safely reach any conclusion about uniqueness of the grounding line position in real ice sheets by studying the singular mathematical solution. A no-slip/ slip singularity on a flat surface is similar to a singularity at the contact line of a drop moving with no slip over a surface. The latter singularity is usually avoided by adopting a slip condition (Equation (2)) near the contact line (Hocking, 1976). Similarly, application of a sliding boundary condition instead of a no-slip condition would remove the singularity, provided that the bottom slope is continuous at the grounding line. If it is not, then the condition

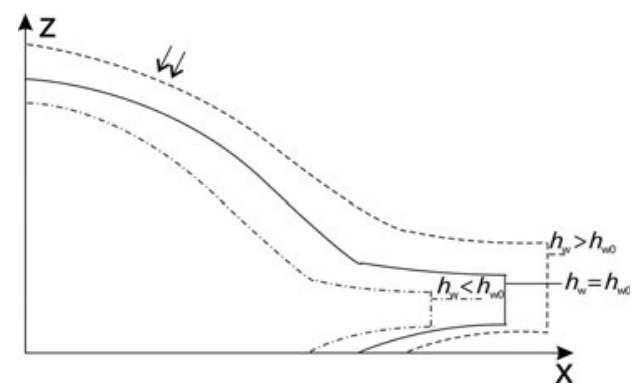

Fig. 3. Different grounding line positions determined by different water depths. The cumulative mass flow rate increases with $x$ due to a constant ice accumulation rate at the ice-sheet surface.

of no vertical motion at the bottom of the grounded ice sheet (Equation (3)) would still give rise to a stress singularity at the grounding line. In order to avoid this complex mathematical issue, we will refer to a problem of elasticity that, firstly, has similar characteristics, and, secondly, allows a much easier experimental understanding of the uniqueness problem.

\section{LIFTING AN ELASTIC SHEET FROM A SUBSTRATE}

Our aim here is to study the effect of condition (3) imposed up to a fixed grounding line position on the solution around the grounding line while other parameters determining external forcing, like the mass flow rate, vary. Let us first note that the rheology of an incompressible linearly elastic material is described as

$$
\tau_{i j}=\frac{E}{2(1+\mu)}\left(\frac{\partial u_{i}}{\partial x_{j}}+\frac{\partial u_{j}}{\partial x_{i}}\right),
$$

where $u_{i}$ and $u_{j}$ are displacements, $E$ is Young's modulus and $\mu$ is Poisson's ratio. This formula is similar to that which describes the rheology of an incompressible linear fluid when one uses velocities, $\mathbf{v}$, instead of displacements, $\mathbf{u}$. The no-slip condition can then be given as

$$
\mathbf{u}=0, \quad z=b,
$$

and the mass balance as

$$
\nabla \cdot \mathbf{u}=0
$$

Therefore, if we consider incompressible elastic deformation under a body force acting along $x$ of an elastic material confined between two horizontal planes with adhesion over the 'upstream' part of the bottom plane and frictionless slip along the rest of the planes, we would derive the same set of equations as those for a linear fluid flow problem with a noslip/slip transition problem, but in terms of displacement, $\mathbf{u}$. This implies the same type of singularity of the stress at a jump between adhesion and free slip when an incompressible elastic material is considered.

Let us now consider the problem of lifting an elastic sheet (e.g. rubber) from a rigid substrate (Fig. 4). One edge of the sheet is fixed, while the other is lifted with a force, $F$, against gravity. We assume no friction at the substrate. The lifting force causes vertical displacement that depends on the force magnitude. The length of the lifted part will be longer for larger lifting forces and shorter for smaller lifting forces. Such behaviour is similar to the ice-sheet length change due to a change in, say, water depth (Fig. 3). 


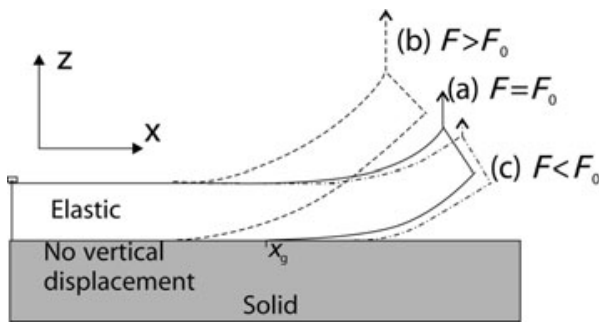

Fig. 4. Schematic of flexure of an elastic sheet on a rigid substrate under a lifting force acting at its edge: (a) a force, $F=F_{0}$; (b) a larger force, $F>F_{0}$; (c) a smaller force, $F<F_{0}$.

We do not aim to find the exact mathematical solution of the problem, but rather appeal to physical experience that could be corroborated by future experimental studies. We apply the same approach as that used in transition zone studies to understand its effect at a physical level.

If we now want to find the position of the lift line, denoted here by $x_{\mathrm{g}}$, for some force, $F_{0}$, and follow the standard method used in modelling the ice-sheet transition zone, then we impose the condition of no vertical motion, Equation (3), up to (as yet unknown) $x_{g}$, preventing the bottom of the sheet from separating from the substrate, while the rest of the bottom is considered to be a free surface. If now the lifting force is varied we can argue, from physical experience, that the condition of no vertical motion, Equation (3), imposed up to the fixed $x_{\mathrm{g}}$ provides multiple solutions of the problem. These solutions are schematically drafted in Figure 5, where the lower surface profiles remind us of those of the ice-shelf solutions in the transition zone shown in Figure 2. Evidently, only one solution presented in Figure 5 corresponds to the natural solutions shown in Figure 4, while the others are artefacts of using Equation (3) within a fixed region (that does not allow the sheet to come off) as well as not considering the full length of the solid substrate (that would not allow the sheet to droop). In these real situations the stress is continuous and the correct solution can be identified from Figure 5 by inspecting the lower surface angle of $0^{\circ}$. The normal stress acting on the substrate is zero at the lift line. The other solutions are described by finite lift angles to ensure the bottom normal stress is finite at the lift line. This is also supported by the theory of peeling at finite local peel angles (Kinloch and Williams, 1994). The presence of a finite angle may lead to a mathematical stress singularity in elastic materials (Kotousov and Lew, 2006), the detailed examination of which is beyond the scope of this work. An important conclusion, however, is that the possible presence of such a mathematical singularity does not change the fact that the only solution of the problem shown in Figure 5 that is a solution of the original problem, shown in Figure 4, is the one possessing a zero lower surface angle.

\section{CONCLUSIONS}

Our conclusion is that the main difficulty in rigorously treating the uniqueness problem of the transition zone solution arises from a mathematical singularity at the grounding line. If continuity is assumed, then a unique relationship between the ice thickness and the integral mass flow rate at the grounding line may be found, if one takes into account the bottom stress while using bottom

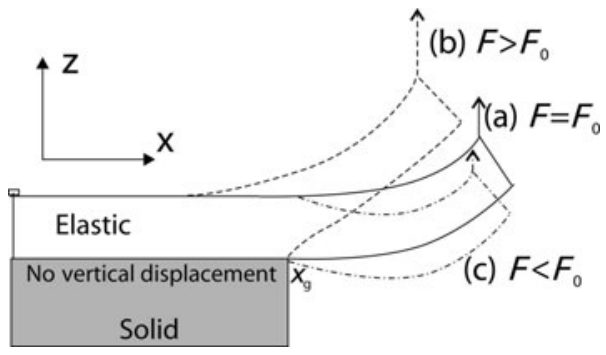

Fig. 5. Schematic of flexure of an elastic sheet on a rigid substrate with no vertical displacement imposed at the bottom, under a lifting force acting at its edge: (a) a force, $F=F_{0}$; (b) a larger force, $F>F_{0}$; (c) a smaller force, $F<F_{0}$. The solid substrate edge is given by the separation point, $x_{\mathrm{g}}$, determined by application of the force $F=F_{0}$.

boundary conditions that prevent vertical motion. This is tantamount to requiring that the sought solution has a zero bottom slope at the grounding line in the floating part. The reason that multiple solutions of the problem are found is that imposing idealized boundary conditions that prevent vertical motion implies the application of an additional force, pulling the grounded ice sheet to the bedrock in the region where the grounded ice sheet is assumed to rest on the bedrock, whatever the other circumstances are. This prevents the ice from naturally retreating or advancing, and allows different mathematical solutions of the transition zone problem; in particular, mathematical solutions with the same thickness but different flow rates can exist. In reality such ice sheets would not be stationary, as the water pressure would be enough for sea water to penetrate beneath the ice, lift the grounded ice sheet and make it retreat. Therefore, in order to choose the physically relevant solution, the vertical stress must be considered at the bottom of the grounded ice sheet and compared to the water pressure. The only situation in this case when there would be no tendency for the ice sheet to either advance or retreat is when the water pressure is equal to the vertical normal stress in the grounded ice sheet at the grounding line. As the grounded ice-sheet bottom is assumed to be horizontal in such simplified studies, with a physical assumption of continuity of the stresses across the grounding line, this is tantamount to requiring the ice-shelf bottom slope to be horizontal at the grounding line (Fig. 2a). With this condition a unique relationship between the ice thickness and the mass flow rate at the grounding line may be found.

\section{REFERENCES}

Barcilon, V. and D.R. MacAyeal. 1993. Steady flow of a viscous ice stream across a no-slip/free-slip transition at the bed. J. Glaciol., 39(131), 167-185.

Chugunov, V.A. and A.V. Wilchinsky. 1996. Modelling of a marine glacier and ice-sheet-ice-shelf transition zone based on asymptotic analysis. Ann. Glaciol., 23, 59-67.

Hindmarsh, R.C.A. 1993. Qualitative dynamics of marine ice sheets. In Peltier, W.R., ed. Ice in the climate system. Berlin, etc., Springer-Verlag, 67-99.

Hocking, L.M. 1976. A moving fluid interface on a rough surface. J. Fluid Mech., 76(4), 801-817.

Kinloch, A.J., C.C. Lau and J.G. Williams. 1994. The peeling of flexible laminates. Int. J. Frac., 66(1), 45-70.

Kotousov, A. and Y.T. Lew. 2006. Stress singularities resulting from various boundary conditions in angular corners of plates of 
arbitrary thickness in extension. Int. J. Solids Struct., 43(17), 5100-5109.

Lestringant, R. 1994. A two-dimensional finite-element study of flow in the transition zone between an ice sheet and an ice shelf. Ann. Glaciol., 20, 67-72.
MacAyeal, D.R. and V. Barcilon. 1988. Ice-shelf response to icestream discharge fluctuations: I. Unconfined ice tongues. J. Glaciol., 34(116), 121-127.

Wilchinsky, A.V. and V.A. Chugunov. 2001. Modelling ice flow in various glacier zones. J. Appl. Math. Mech., 65(3), 479-493.

MS received 18 August 2006 and accepted in revised form 24 February 2007 